\title{
pH-sensitive Beschichtung für SPR-basierte Sensoren
}

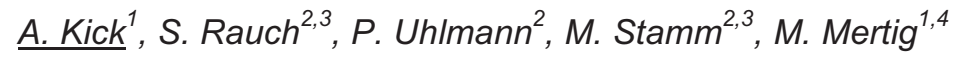 \\ ${ }^{1}$ Kurt-Schwabe-Institut für Mess- und Sensortechnik e.V. Meinsberg, Kurt-Schwabe-Str. 4, 04736 \\ Waldheim, Deutschland \\ Michael.Mertig@tu-dresden.de \\ ${ }^{2}$ Leibniz-Institut für Polymerforschung Dresden e.V., Hohe Str. 6, 01069 Dresden, Deutschland \\ ${ }^{3}$ Professur für Physikalische Chemie Polymerer Materialien, Technische Universität Dresden, 01062 \\ Dresden, Deutschland \\ ${ }^{4}$ Professur für Physikalische Chemie, Mess- und Sensortechnik, Technische Universität Dresden, \\ 01062 Dresden, Deutschland
}

\begin{abstract}
:
Neue Ansätze für die optische Bestimmung des pH-Wertes in Bioreaktoren sind von wachsendem Interesse. Es werden $\mathrm{pH}$-sensitive Beschichtungen entwickelt, welche die Detektion der pH-Werte im sauren Bereich erlauben. Dabei soll das Prinzip der Oberflächenplasmonenresonanz (SPR) genutzt werden, um eine empfindliche, schnelle und reproduzierbare Messung zu ermöglichen. Mit der SPRMethode werden Änderungen der Brechzahl im oberflächennahen Medium an der Sensoroberfläche hoch empfindlich registriert. Es ist ein optisches Verfahren.
\end{abstract}

Schlüsselwörter: pH-sensitiv, SPR, Sensor, Poly(2-vinylpyridin), Poly(glycidylmethacrylat)

\section{Einleitung}

Neue Ansätze für die optische Bestimmung des $\mathrm{pH}-$ Wertes in Bioreaktoren sind von wachsendem Interesse. Das Ziel dieser Arbeit ist es, eine $\mathrm{pH}$-sensitive Beschichtung zu entwickeln, welche die Detektion der $\mathrm{pH}$-Werte im sauren Bereich erlaubt. Dabei soll das Prinzip der Oberflächenplasmonenresonanz (SPR) genutzt werden, um eine empfindliche, schnelle und reproduzierbare Messung zu ermöglichen.

Mit der SPR-Methode werden Änderungen der Brechzahl im oberflächennahen Medium an der Sensoroberfläche hoch empfindlich registriert. Es ist ein optisches Verfahren [1]. Die Sensoroberfläche besteht aus einem $50 \mathrm{~nm}$ dicken Goldfilm. Wird die Goldoberfläche mit einem $\mathrm{pH}$-sensitiven Polymer beschichtet, kann seine Protonierung mittels SPR beobachtet werden. Dabei können das Quellen des Polymers und die lonenadsorption diskutiert werden $[2,3]$.

\section{SPR-Chip und SPR-Spektrometer}

Es wurden optisch transparente SPR-Chips (76 $\mathrm{mm} \times 26 \mathrm{~mm} \times 4 \mathrm{~mm}$ ) aus einem Cyclo-
Olefin-Copolymer mit integrierten optischen Linsen und einer $50 \mathrm{~nm}$ dicken Goldschicht $(3 \mathrm{~mm} \times 12 \mathrm{~mm})$ verwendet. Das SPRSpektrometer erlaubt eine örtlich aufgelöste Untersuchung der Sensoroberfläche. Eine CCD-Kamera dient zur winkelaufgelösten Analyse der SPR. Das SPR-Signal, angegeben in Pixel, ist die Verschiebung des Minimums des reflektierten Lichtes im Winkelspektrum [4,5].

\section{Schichtaufbau}

In Bild 1 ist der schematische Aufbau der Sensoroberfläche gezeigt. Auf die Goldoberfläche wird zunächst eine selbstorganisierende Monoschicht (SAM) der 3-Mercaptopropionsäure aufgebracht. Danach wird Poly(glycidylmethacrylat) (PGMA, ein Polymer mit reaktiven Glycidylgruppen) aus Aceton aufgetragen $\left(130^{\circ} \mathrm{C}\right.$, Vakuumofen). Abschließend wird das $\mathrm{pH}$ sensitive Polymer Poly(2-vinylpyridin) (P2VP, mit zur terminalen Carboxylgruppen) aus Aceton auf die PGMA-Schicht aufgebracht ( $5 \mathrm{~h}$, $130^{\circ} \mathrm{C}$, Vakuumofen). 


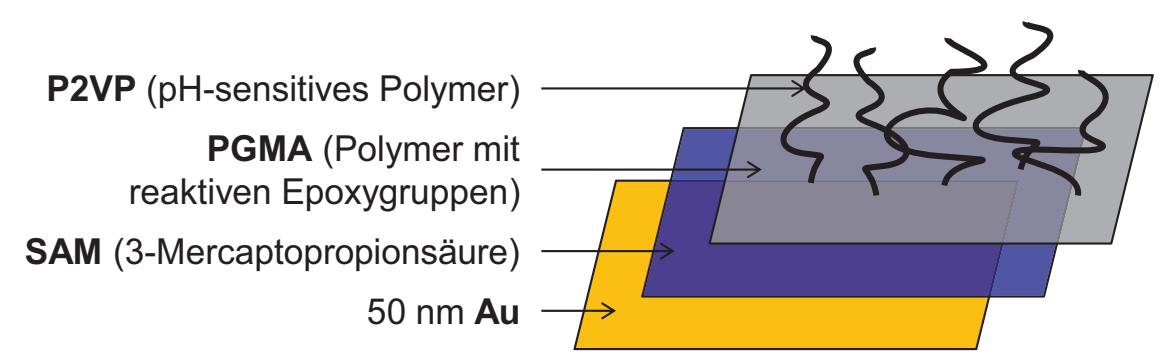

Bild 1. Schichtaufbau der Sensoroberfläche; P2VP - Poly-2-vinylpyridin mit terminaler Carboxylgruppe; PGMA - Poly(glycidylmethacrylat).

\section{SPR-Messung}

Bei der Messung werden die SPR-Signale in Abhängigkeit von der SalzsäureKonzentrationen bzw. der pH-Werte in Wasser aufgezeichnet. Es werden die SPR-Signale auf dem $\mathrm{pH}$-sensitiven P2VP und auf der Referenz (PGMA) ausgewertet. Lösungen mit aufsteigendem und danach mit abfallendem $\mathrm{pH}$-Wert werden über die Sensoroberfläche mittels Mikrofluidik gepumpt. Das SPR-Signal in Wasser wird auf 0 Pixel gesetzt. In Bild 2 sind die referenzierten Signale als Funktion des $\mathrm{pH}$-Wertes einer Salzsäurelösung gezeigt. Man erhält eine schnelle und deutliche Antwort innerhalb weniger Sekunden. Außerdem ist keine Hysterese zu erkennen und die Messwerte sind sehr gut reproduzierbar.

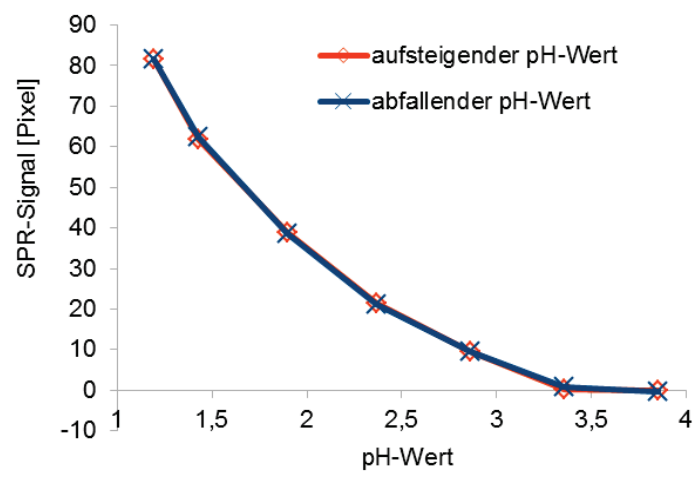

Bild. 2. Differenz der SPR-Signale des P2VP und der Referenzfläche (PGMA). Dargestellt sind die $S P R$-Signale, gemessen für aufsteigende und abfallende $\mathrm{pH}$-Werte.

\section{Ausblick}

In zukünftigen Experimenten soll die Selektivität des $\mathrm{pH}$-Wert-Sensors in Anwesenheit unterschiedlicher lonen untersucht werden. Außerdem soll getestet werden, ob Goldnanopartikel an das P2VP angebunden werden können, um die Empfindlichkeit des SPR-Sensors zu steigern [6].

\section{Danksagung}

Diese Arbeit wird gefördert durch das Sächsische Ministerium für Wissenschaft und Kunst.

\section{Literatur}

[1] J. Homola, S. Yee, G. Gauglitz, Surface Plasmon Resonance Sensors: Review, Sensors and Actuators B: Chemical 54 (1-2), 3-15 (1999), DOI: 10.1016/S0925-4005(98)00321-9

[2] I. Tokareva, S. Minko, J. H. Fendler, E. Hutter, Nanosensors Based on Responsive Polymer Brushes and Gold Nanoparticle Enhanced Transmission Surface Plasmon Resonance Spectroscopy, Journal of the American Chemical Society 126 (49), 15950-15951 (2004), DOI: $10.1021 / \mathrm{ja} 044575 \mathrm{y}$

[3] X. Wang, G. Liu, G. Zhang, Conformational Behavior of Grafted Weak Polyelectrolyte Chains: Effects of Counterion Condensation and Nonelectrostatic Anion Adsorption, Langmuir 27 (16), 9895-9901 (2011), DOI: 10.1021/la201057h

[4] N. Danz, A. Hofmann, Plasmon Resonance Sensor, WO 2008/025488

[5] N. Danz, A. Kick, F. Sonntag, S. Schmieder, B. Höfer, U. Klotzbach, M. Mertig, Surface Plasmon Resonance Platform Technology for MultiParameter Analyses on Polymer Chips, Engineering in Life Sciences 11 (6), 566-572 (2011), DOI: 10.1002/elsc.201000192

[6] R. Contreras-Caceres, C. Dawson, P. Formanek, D. Fischer, F. Simon, A. Janke, P. Uhlmann, M. Stamm, Polymers as Templates for $\mathrm{Au}$ and Au@Ag Bimetallic Nanorods: UV-Vis and Surface Enhanced Raman Spectroscopy, Chemistry of Materials 25 (2), 158-169 (2013), DOI: $10.1021 / \mathrm{cm} 303132$ 